\title{
Duality in the non-relativistic harmonic oscillator quark model in the Shifman-Voloshin limit : a pedagogical example
}

\author{
A. Le Yaouanc ${ }^{a}$, D. Melikhov ${ }^{b}$ 巴, V. Morenas ${ }^{c}$, L. Oliver ${ }^{a}$, O. Pène ${ }^{a}$ and J.-C. Raynal ${ }^{a}$ \\ ${ }^{a}$ Laboratoire de Physique Théorique, Université de Paris XI, \\ Bâtiment 210, 91405 Orsay Cedex, Francę \\ ${ }^{b}$ Institut für Theoretische Physik, Universität Heidelberg, \\ Philosophenweg 16, D-69120, Heidelberg, Germany \\ ${ }^{c}$ Laboratoire de Physique Corpusculaire, Université Blaise Pascal - CNRS/IN2P3, \\ 63000 Aubière Cedex, France
}

\begin{abstract}
The detailed way in which duality between sum of exclusive states and the free quark model description operates in semileptonic total decay widths, is analysed. It is made very explicit by the use of the non relativistic harmonic oscillator quark model in the SV limit, and a simple interaction current with the lepton pair. In particular, the Voloshin sum rule is found to eliminate the mismatches of order $\delta m / m_{b}^{2}$.
\end{abstract}

LPT-Orsay 00-29

HD-THEP-00-14

PCCF RI 00-10

hep-ph/0005039

\footnotetext{
${ }^{1}$ Alexander-von-Humboldt fellow. On leave from Nuclear Physics Institute, Moscow State University, Moscow, Russia

${ }^{2}$ Unité Mixte de Recherche - CNRS - UMR 8627
} 


\section{Introduction}

Discussions have recently arisen about the possibility that expectations from OPE for some types of semi-leptonic rates may be violated by terms of order $1 / m_{Q}$. The argument of Nathan Isgur [1] is founded on general considerations ; namely the duality is obtained in the infinite mass limit through cancellation between the falloff of the ground state contribution and the rise of the excitations (the Bjorken sum rule indeed relates the derivatives of these contributions with respect to $w$, near $w=1$ ), but at finite mass there is some mismatch near zero recoil, which could be of order $1 / m_{Q}$. Indeed, in terms of $t$, the quadri-dimensional transferp:

$$
t=\left(q^{0}\right)^{2}-\vec{q}^{2},
$$

the respective $t_{\max }$ do not coincide anymore. The argument is then given by the author further likeliness by some calculations within a very simple "toy" model : the non relativistic harmonic oscillator (HO) potential model.

In the present letter, we will not discuss directly the issue about QCD (see our article [3]). We simply stick to the very model used in [1], and show that within this model, calculating the total integrated rate $\Gamma_{\text {inclusive }}$ by summation on the relevant final (bound) states, duality with free quark decay rate is in fact satisfied, in the SV (ShifmanVoloshin[2]) limitf; this means that the difference $\Gamma_{\text {inclusive }}-\Gamma_{\text {free quark }}$ comes out as expected, which implies in particular (as discussed below) cancellation of terms of relative order $(\delta m)^{2} / m_{b}^{2}$ and $\delta m / m_{b}^{2}$ (by relative, we mean with respect to the free quark decay rate ; note that such terms correspond to $\left(1 / m_{Q}\right)^{0},\left(1 / m_{Q}\right)^{1}$ in the usual $1 / m_{Q}$ expansion). Our argument is for integrated decay rates, so we do not claim anything on possible effects in differential or partially integrated rates. Also, of course, we cannot exclude by such argument that something odd may happen in QCD.

One very interesting point raised in the discussion of [1] is about the very specific cancellations which are necessary for duality to hold, and about the contributions of the various regions of phase space. We try to analyze through our demonstration how such cancellations occur in subleading order for total widths. An interesting consequence of the analysis is that to find the required cancellations, one needs not to consider only the sum rule of Bjorken ; one has to take into account in addition the Voloshin sum rule (the fact that one needs the sum rules has been suggested by the Minnesota group in their discussion with N. Isgur [1], but is made here quite explicit; for related discussions in QCD by the same group, see [4]). In fact, the Voloshin sum rule is exactly what is needed for cancellation of terms of relative order $\delta m / m_{b}^{2}$ in the difference $\Gamma_{\text {inclusive }}-\Gamma_{\text {free quark }}$. The sum rules are trivially satisfied in the $\mathrm{HO}$ model, but it is not so trivial in general. Our conclusion is not in contradiction with the mismatch occuring near zero recoil, considered in [1], because the latter is very small parametrically with respect to the terms we consider in the total width.

Note that the use of SV limit is not essential to demonstrate duality in this way, and neither is the use of an HO potential. Their choice is pedagogical. Indeed we have

\footnotetext{
${ }^{3} t$ is we use the old standard notation $t$, to avoid confusion with the tridimensional $|\vec{q}|^{2}$, which will be used extensively in this non relativistic (NR) context.

${ }^{4}$ By SV limit, we do not mean simply that the recoil velocity is small, but also, as in the original paper [2], that $\frac{\delta m}{m}$ is small ; in addition, $\delta m$ is taken large with respect to light quark parameters ; in non relativistic quantum mechanics, we assume : $\Delta=\frac{1}{m_{d} R^{2}} \ll \delta m$ and $m_{d}, \delta m \ll m_{b}, m_{c}, \Delta$ being the level spacing.
} 
also done the demonstration for an arbitrary potential ( [5]) and also for fixed $m_{c} / m_{b}$ ratio. Nevertheless, the particular case considered here is of pedagogical interest, because on the one hand the discussion in the SV limit is much simpler, and the similar discussion in QCD can hardly be made beyond the SV limit, and because on the other hand, within HO model, we can give explicit expressions. Moreover, we are able to give a complete proof that in the HO model $1 / m_{Q}$ terms are absent in the ratio $\Gamma_{\text {inclusive }} / \Gamma_{\text {free quark }}$ beyond the SV limit (article to appear [6]). Note also that the demonstration is independent of the leptonic tensor, as we have also shown elsewhere, but we choose here one specific for illustration. On the other hand, the coefficient of the terms of order $\frac{1}{R^{2} m_{b}^{2}}$, which we also evaluate, is model-dependent (in particular it depends on the choice of the leptonic tensor; we choose here one for illustration).

\section{Model}

- The model for hadrons is the non relativistic harmonic oscillator quark model (the motion of quarks both internal and due to overall hadron are both treated non relativistically), describing the initial (quarks $b$ and $d$ ) and final ( $c$ and $d$ ) hadrons. The potential is assumed to be flavor independent, which is crucial for the demonstration. The great advantage of the harmonic oscillator, which appears in the summation on final states, is that very few states contributes to the transition rates in the limited expansion in 1overm $_{b}$ which we perform (see next section). Energy levels, for a state labelled by $\left(n_{x}, n_{y}, n_{z}\right), n=n_{x}+n_{y}+n_{z}$, write :

$$
E_{n}=m_{b, c}+m_{d}+\left(\frac{3}{2}+n\right) \frac{1}{\mu_{b, c} R_{b, c}^{2}}
$$

where $\mu_{b, c}$ are the reduced masses $\frac{m_{b, c} m_{d}}{m_{b, c}+m_{d}}$ and the radii $R_{b, c}^{2}$ can be written as :

$$
R_{b, c}^{2}=\sqrt{\frac{m_{d}}{\mu_{b, c}}} R_{\infty}^{2}
$$

$R_{\infty}$ being the radius in the infinite mass limit. We will often denote the first level excitation energy in the infinite mass limit as :

$$
\Delta=\frac{1}{m_{d} R_{\infty}^{2}} .
$$

For simplicity, from now on, we denote :

$$
R_{\infty}=R
$$

- Quarks are then coupled to lepton pairs : $b \rightarrow c \ell \nu$, through a quark vector current $j^{0}=1, \vec{j}=0$ f (or equivalently we can speak of spinless quarks), and a leptonic tensor, which will be described by functions denoted generically through letter $L$ and some

\footnotetext{
${ }^{5}$ Note that we do not claim to make a systematic non relativistic expansion of a relativistic theory, but only to consider a non relativistic Hamiltonian for the bound states; we can choose freely the weak interaction current. The essential point is then to treat consistently the matrix elements according to the chosen interactions, in the specified SV expansion.
} 
arguments and indices. $\vec{P}$ and $\vec{P}^{\prime}$ are the initial and final hadron momenta ; the total energies of hadrons are $P^{0}=E+\vec{P}^{2} / 2\left(m_{b}+m_{d}\right), P^{\prime 0}=E^{\prime}+\vec{P}^{\prime 2} / 2\left(m_{c}+m_{d}\right)$, with $E, E^{\prime}$ the energies at rest ; but, in practice, we will always work in the initial hadron rest frame : $\vec{P}=0 ; \vec{P}$ and $\overrightarrow{P^{\prime}}$ are the initial and final quark momenta. We denote $\vec{q}=\vec{P}-\overrightarrow{P^{\prime}}=-\overrightarrow{P^{\prime}}$. The basic equation is then, in the initial state rest frame:

$$
\Gamma_{\text {inclusive }}=K \sum_{n} \int_{0}^{|\vec{q}|_{\max , n}} d|\vec{q}||\vec{q}|^{2} L_{n}(|\vec{q}|) \sum_{n=n_{x}+n_{y}+n_{z}}\left|j_{0 \rightarrow\left(n_{x}, n_{y}, n_{z}\right)}\right|^{2}
$$

The constant $K$ depends only on the decay interaction strength. The constant $K$ will be ommitted in the rest of the letter. $\sum_{n=n_{x}+n_{y}+n_{z}}\left|j_{0 \rightarrow\left(n_{x}, n_{y}, n_{z}\right)}\right|^{2}$ only depends on $|\vec{q}|$. The angular integration has been performed. The notations $L_{n}(|\vec{q}|)$ and $|\vec{q}|_{\text {max }, n}$ are explained now. A priori, after angular integration, the leptonic tensor appears through a function of energy loss $q^{0}$ and $\vec{q}^{2}, L\left(q^{0},|\vec{q}|^{2}\right)$. However, for the decay from the ground state to a h.o. state labelled by $\left(n_{x}, n_{y}, n_{z}\right)$, by energy conservation, $q^{0}=P^{0}-P^{\prime 0}$ is just a function of $|\vec{q}|$ and $\left(n_{x}, n_{y}, n_{z}\right)$. Moreover, the energy loss $q^{0}$ will depend only on $n=n_{x}+n_{y}+n_{z}$. and we then denote as $L_{n}(|\vec{q}|)$ the result of $L\left(q^{0},|\vec{q}|^{2}\right)$, when the energy loss $q^{0}$ is assumed to be calculated for the corresponding $n$, as a function of $|\vec{q}|$. Indeed, for a state with degree of excitation $n$ :

$$
q^{0}(n,|\vec{q}|)=m_{b}-m_{c}+\frac{3}{2}\left(\frac{1}{\mu_{b} R_{b}^{2}}-\frac{1}{\mu_{c} R_{c}^{2}}\right)-n \frac{1}{\mu_{c} R_{c}^{2}}-\frac{|\vec{q}|^{2}}{2\left(m_{c}+m_{d}\right)} .
$$

Now $q_{\text {max }}$ is determined by the equation $t=\left(q^{0}\right)^{2}-|\vec{q}|^{2}=0, q^{0}(|\vec{q}|)=|\vec{q}|$ :

$$
|\vec{q}|_{\max , n}=\frac{2\left(m_{c}+m_{d}\right)(\delta E)_{n}}{2\left(m_{c}+m_{d}\right)+\sqrt{\left(m_{c}+m_{d}\right)^{2}+2\left(m_{c}+m_{d}\right)(\delta E)_{n}}}
$$

where

$$
(\delta E)_{n}=q^{0}(n, \vec{q}=0)=m_{b}-m_{c}+\frac{3}{2}\left(\frac{1}{\mu_{b} R_{b}^{2}}-\frac{1}{\mu_{c} R_{c}^{2}}\right)-n \frac{1}{\mu_{c} R_{c}^{2}} .
$$

$|\vec{q}|_{\text {max }}$ just depends on $n . L\left(q^{0},|\vec{q}|^{2}\right)$ can be taken as an arbitrary function without spoiling any of the general statements made below, but for definiteness we will henceforth choose :

$$
L\left(q^{0}, \vec{q}^{2}\right)=3\left(q^{0}\right)^{2}-|\vec{q}|^{2},
$$

inspired by a static quark approximation of the $\mathrm{V}$-A current. The corresponding free quark decay rate is :

$$
\Gamma_{\text {free }}=K \int_{0}^{|\vec{q}|_{\text {max }, \text { free }}} d|\vec{q}||\vec{q}|^{2} L\left(q^{0},|\vec{q}|^{2}\right)
$$

with :

$$
\begin{gathered}
q^{0}(\text { free },|\vec{q}|)=m_{b}-m_{c}-\frac{|\vec{q}|^{2}}{2 m_{c}} \\
|\vec{q}|_{\text {max }, \text { free }}=\frac{2 m_{c} \delta m}{\left(m_{c}+\sqrt{m_{c}^{2}+2 m_{c} \delta m}\right)}
\end{gathered}
$$

with $\delta m=m_{b}-m_{c}$. 


\section{SV expansion and demonstration of duality}

- We have then to consider the expansion of

$$
\epsilon=\frac{\Gamma_{\text {inclusive }}-\Gamma_{\text {free }}}{\Gamma_{\text {free }}}
$$

in powers of $\frac{1}{m_{b}}$, and the aim is in principle to show that it begins with order $\frac{1}{m_{b}^{2}}$ only, as expected from a formal OPE (the NR version of OPE will be explained in the more developped article). More precisely this holds in the limit $m_{b} \rightarrow \infty$ with $r=\frac{m_{c}}{m_{b}}$ fixed, for which we reserve for clarity the term " usual $1 / m_{Q}$ expansion". However, we will work in the SV (Shifman-Voloshin) limit, which corresponds to making in addition an expansion in $1-r$. Namely, with :

$$
\delta m=m_{b}-m_{c}
$$

we write $m_{c}=m_{b}-\delta m$ and we expand in powers of $\frac{1}{m_{b}}$, keeping $\delta m$ fixed, as well as the light quark parameters, $m_{d}, 1 / R$; then, we make a second limited expansion, taking $\Delta=\frac{1}{m_{d} R^{2}}$ small with respect to $\delta m$. The terms have the form $\frac{(\delta m)^{k^{\prime}}}{\left(m_{b}\right)^{k}}$ times light quark factors. But then the aim must be more than just showing the absence of powers $\frac{1}{\left(m_{b}\right)^{k}}$, $k<2$ in $\epsilon$.

Indeed, if it is true, this would not in principle preclude terms of the type $\frac{(\delta m)^{k^{\prime}}}{m_{b}^{2}}$ $\left(k^{\prime}>0\right)$ in $\epsilon$. Such terms would be large in practice, since $\delta m$ is not so small. And in fact, they would correspond, in terms of the usual $1 / m_{Q}$ expansion, to terms of order $\left(1 / m_{Q}\right)^{0},\left(1 / m_{Q}\right)$, since $\delta m$ would be then $\propto m_{Q}$. Such terms are not expected from OPE. We must therefore show that such terms do not exist in the final result, and we show it in fact. More precisely, we show that potentially large terms of the type $\frac{(\delta m)^{2}}{m_{b}^{2}}$, $\frac{m_{d} \delta m}{m_{b}^{2}}$, which appear in particular contributions, do finally cancel out, leaving us with terms of the type $\frac{1}{R^{2} m_{b}^{2}}$ (terms with $k^{\prime}>2$ simply do not appear in the way we calculate $\epsilon$, neither do terms with power $\frac{1}{\left(m_{b}\right)^{0}}$ or $\frac{1}{m_{b}}$ - in fact, the delicate part consists in showing the cancellation of $\frac{m_{d} \delta m}{m_{b}^{2}}$ terms). This is all that is required by duality with free quarks, as concerns the terms with power $\left(\frac{1}{m_{b}}\right)^{k}, k \leq 2$. We will calculate the terms of type $\frac{1}{R^{2} m_{b}^{2}}$, which do not vanish in general. Note that such terms are small with respect to $\frac{m_{d} \delta m}{m_{b}^{2}}$ by a factor $\frac{\Delta}{\delta m}$. In the usual $1 / m_{Q}$ expansion they correspond to order $1 / m_{Q}^{2}$. On the other hand, we will not calculate in the expansion of $\epsilon$ smaller terms having also the power $\frac{1}{m_{b}^{2}}$, but which contain still additional powers of $\frac{\Delta}{\delta m}$ with respect to $\frac{1}{R^{2} m_{b}^{2}}$, corresponding in $\Gamma_{\text {inclusive }}-\Gamma_{\text {free }}$ to terms like $\frac{(\delta m)^{4} \times \Delta}{m_{b}^{2}}, \frac{(\delta m)^{3} \times \Delta^{2}}{m_{b}^{2}}$, etc... and retain only the terms proportional to $\Gamma_{\text {free }} \propto(\delta m)^{5}$. The neglected terms correspond to terms of relative order $1 / m_{Q}^{3}$ or beyond in the $1 / m_{Q}$ expansion. For sake of simplicity, we will neither examine further checks of duality in terms of the type $\frac{(\delta m)^{k^{\prime}}}{\left(m_{b}\right)^{k}}$, with $k>2$.

In any case, we see that we do have to calculate terms with a power $\frac{1}{m_{b}^{2}}$ and not $\frac{1}{m_{b}}$ only, since the terms with a power $\frac{1}{m_{b}^{2}}$ may correspond to terms of the order

\footnotetext{
${ }^{6}$ Note that, in the present letter, we term generically as power $\frac{1}{\left(m_{b}\right)^{k}}$ all the terms which contain the factor $\frac{1}{\left(m_{b}\right)^{k}}$, whatever the powers of $\delta m$ and light quantities.
} 
$\left(1 / m_{Q}\right)^{0},\left(1 / m_{Q}\right)^{1}$ in the usual expansion. The method precisely consists in writing the difference $\Gamma_{\text {inclusive }}-\Gamma_{\text {free }}$ as a sum of terms which contain already a power $\frac{1}{m_{b}^{2}}$, and then to demonstrate the above additional cancellations.

- The advantage of harmonic oscillator (HO) model is that the level $n=1$ (which corresponds to $L=1$ states) appears only with a power $\frac{1}{m_{b}^{2}}$, and that higher levels come only with a power $\frac{1}{m_{b}^{3}}$ at least. Since we keep terms with a power $\frac{1}{m_{b}^{2}}, i \leq 2$, we only need consider $n=0,1$ states. For sake of simplicity, we denote their respective contributions $\Gamma_{0,1}$.

We have at this order, by expanding the matrix elements :

$$
\Gamma_{0} \simeq \int_{0}^{|\vec{q}|_{\max , 0}} d|\vec{q}||\vec{q}|^{2} L_{n=0}(|\vec{q}|)\left(1-\rho^{2} \frac{|\vec{q}|^{2}}{m_{b}^{2}}\right),
$$

where $\rho^{2}=\frac{1}{2} m_{d}^{2} R^{2}$ is the standard slope of the ground state form factor with respect to $w\left(w-1 \simeq \frac{1}{2} \frac{|\vec{q}|^{2}}{m_{b}^{2}}\right)$; note that effect of non complete overlapping between hadrons with $b$ and $c$ quarks is completely negligible here, since it contributes at order $1 / R^{2} \frac{(\delta m)^{2}}{m_{b}^{4}}$. For $L=1$ states:

$$
\Gamma_{1} \simeq \int_{0}^{|\vec{q}|_{\max , 1}} d|\vec{q}||\vec{q}|^{2} L_{n=1}(|\vec{q}|) \tau^{2} \frac{|\vec{q}|^{2}}{m_{b}^{2}}
$$

with $\tau=\frac{m_{d} R}{\sqrt{2}}$ corresponding to the $\tau_{1 / 2,3 / 2}(w=1) \times \sqrt{3}$. The other excitations do not contribute at this order, because the matrix element $\langle n|\vec{r}| 0>$ is non zero only if $n=1$. From the explicit expressions, we have the relations :

$$
\begin{aligned}
& \rho^{2}-\tau^{2}=0, \\
& \Delta \tau^{2}=\frac{m_{d}}{2},
\end{aligned}
$$

( $\Delta$ being the level spacing, (1) as non relativistic analogues of the Bjorken and Voloshin sum rules. These sum rules could then be used to generalise the present analysis.

In fact, we will try as much as possible not to specify separately $\Delta, \rho, \tau$, but to use only the above sum rules and expressions for $\Gamma_{0,1}$.

- The strategy is to note that the difference between $\Gamma_{0}+\Gamma_{1}$ and $\Gamma_{\text {free }}$ can be reexpressed by successive steps :

1) Decomposition into the same difference with $L_{0,1}(|\vec{q}|)$ substituted by their free counterpart $L_{\text {free }}(|\vec{q}|)$ (contribution (I)) plus a $\frac{1}{m_{b}^{2}}$ term (contribution (II)). 2) Then the first contribution (I) is rewritten trivially as a difference between two contributions having a power $\frac{1}{m_{b}^{2}}$ relative to the free quark decay integrand, further shown to be of relative order $\frac{1}{R^{2} m_{b}^{2}}$ or smaller. 3) It is also shown that in contribution (II), which contains manifestly a power $\frac{1}{m_{b}^{2}}$, there are only terms of the type $\frac{1}{R^{2} m_{b}^{2}}$ or smaller.

- In a first step, using the respective expressions given above for the $q^{0}(|\vec{q}|)$ 's, and expanding it to the required order, we find :

$$
q^{0}(\text { free },|\vec{q}|) \simeq \delta m-\frac{|\vec{q}|^{2}}{2 m_{c}}
$$




$$
\begin{gathered}
q^{0}(n=0,|\vec{q}|) \simeq \delta m\left(1-\frac{3}{4 m_{b}^{2} R^{2}}\right)-\frac{|\vec{q}|^{2}}{2\left(m_{c}+m_{d}\right)} \\
q^{0}(n=1,|\vec{q}|) \simeq \delta m-\Delta
\end{gathered}
$$

Note that in our expansion, the main term in the three quantities is $\delta m$. The main term of $q_{\max }$ is then also $\delta m\left(q_{\max }=q^{0}\right.$ at $\left.t=0\right)$. We use these expansions to make, in the integrals for $\Gamma_{0,1}$ :

$$
\begin{gathered}
L_{0}(|\vec{q}|) \simeq L_{\text {free }}(|\vec{q}|)+6 \delta m\left(-\frac{3 \delta m}{4 R^{2} m_{b}^{2}}+\frac{m_{d}|\vec{q}|^{2}}{2 m_{b}^{2}}\right) \\
L_{1}(|\vec{q}|) \simeq L_{\text {free }}(|\vec{q}|)+6 \delta m(-\Delta)+3 \Delta^{2}
\end{gathered}
$$

The second terms in the r.h.s. come from the difference between the respective $q^{0}$, as a function of $|\vec{q}|$. Note that in the expansion of $L_{1}(|\vec{q}|)$, one can neglect terms in $\frac{1}{m_{b}^{2}}$ because $\Gamma_{1}$ has already a power $\frac{1}{m_{b}^{2}}$. We get $\Gamma_{\text {inclusive }}-\Gamma_{\text {free }} \simeq \delta \Gamma_{I}+\delta \Gamma_{I I}$ with :

$$
\begin{aligned}
\delta \Gamma_{I}= & \int_{0}^{|\vec{q}|_{\text {max }, 0}} \quad d|\vec{q}||\vec{q}|^{2} L_{\text {free }}(|\vec{q}|)\left(1-\rho^{2} \frac{|\vec{q}|^{2}}{m_{b}^{2}}\right)+ \\
& \int_{0}^{|\vec{q}|_{\text {max }, 1}} \quad d|\vec{q}||\vec{q}|^{2} L_{\text {free }}(|\vec{q}|) \tau^{2} \frac{|\vec{q}|^{2}}{m_{b}^{2}}- \\
& \int_{0}^{|\vec{q}|_{\text {max }, \text { free }}} \quad d|\vec{q}||\vec{q}|^{2} L_{\text {free }}(|\vec{q}|)
\end{aligned}
$$

and

$$
\begin{aligned}
\delta \Gamma_{I I}= & \int_{0}^{|\vec{q}|_{\text {max }, 0}} \quad d|\vec{q}||\vec{q}|^{2} 6 \delta m\left(-\frac{3 \delta m}{4 R^{2} m_{b}^{2}}+\frac{m_{d}|\vec{q}|^{2}}{2 m_{b}^{2}}\right)+ \\
& \int_{0}^{|\vec{q}|_{\text {max }, 1}} \quad d|\vec{q}||\vec{q}|^{2}\left(6 \delta m(-\Delta)+3 \Delta^{2}\right)\left(\tau^{2} \frac{|\vec{q}|^{2}}{m_{b}^{2}}\right) .
\end{aligned}
$$

- Contribution I. One can write it as the difference of two integrals which have already manifestly a factor $\frac{1}{m_{b}^{2}}$, i.e. the terms with power $\frac{1}{\left(m_{b}\right)^{0}}$ or $\frac{1}{m_{b}}$ are already cancelled (this amounts to using $\rho^{2}-\tau^{2}=0$, which is the particular form of the Bjorken sum rule in the model):

$$
\begin{aligned}
\delta \Gamma_{I}= & \int_{|\vec{q}|_{\text {max }, 0 \text { free }}}^{|\vec{q}|_{\text {max }}} \quad d|\vec{q}||\vec{q}|^{2} L_{\text {free }}(|\vec{q}|)- \\
& \int_{q_{\text {max }, 1}}^{|\vec{q}|_{\text {max }, 0}} \quad d|\vec{q}||\vec{q}|^{2} L_{\text {free }}(|\vec{q}|) \tau^{2} \frac{|\vec{q}|^{2}}{m_{b}^{2}}
\end{aligned}
$$

We first expand each integral. - One has :

$$
|\vec{q}|_{\max , 0}-|\vec{q}|_{\max , \text { free }} \simeq \delta m\left(\frac{1}{2} \frac{m_{d} \delta m}{m_{b}^{2}}-\frac{3}{4} \frac{1}{R^{2} m_{b}^{2}}\right),
$$

whence

$$
\int_{|\vec{q}|_{\text {max }, \text { free }}}^{|\vec{q}|_{\text {max }, 0}} d|\vec{q}||\vec{q}|^{2} L_{\text {free }}(|\vec{q}|) \simeq \delta m\left(\frac{1}{2} \frac{m_{d} \delta m}{m_{b}^{2}}-\frac{3}{4} \frac{1}{R^{2} m_{b}^{2}}\right)(\delta m)^{2} L_{\text {free }}\left(|\vec{q}|_{\text {max }}=\delta m\right) .
$$


One can make $|\vec{q}| \simeq \delta m$ in the integrand, because the integration interval contains already a power $1 / m_{b}^{2}$, and the difference between $|\vec{q}|$ and $\delta m$ contains a further $1 / m_{b}$ factor.

- The second integral is more delicate, because the integration interval has not a factor $1 / m_{b}^{2}$; it is just $\simeq \Delta$; the variation of $|\vec{q}|$ is not negligible. One must do a limited expansion of the integrand in powers of $\frac{\Delta}{\delta}$, so as to retain at least terms of the type $\frac{1}{R^{2} m_{b}^{2}}$. It is there that the second expansion,in powers of $\frac{\Delta}{\delta}$, enters the game :

$$
\begin{array}{ll}
\int_{|\vec{q}|_{\text {max }, 1}}^{|\vec{q}|_{\text {max }, 0}} & d|\vec{q}||\vec{q}|^{2} L_{\text {free }}(|\vec{q}|) \tau^{2} \frac{|\vec{q}|^{2}}{m_{b}^{2}} \simeq \\
\int_{\delta m-\Delta}^{\delta m} & d|\vec{q}||\vec{q}|^{2} L_{\text {free }}(|\vec{q}|) \tau^{2} \frac{|\vec{q}|^{2}}{m_{b}^{2}} \simeq \\
& \Delta(\delta m)^{2} L_{\text {free }}(|\vec{q}|=\delta m) \tau^{2} \frac{(\delta m)^{2}}{m_{b}^{2}}- \\
& \frac{\Delta^{2}}{2} \frac{\tau^{2}}{m_{b}^{2}} \frac{d}{d|\vec{q}|}\left(|\vec{q}|^{4} L_{\text {free }}(|\vec{q}|)\right)(|\vec{q}|=\delta m) .
\end{array}
$$

Let us note that to estimate the relative order of the different terms, one has to divide by a reference rate, which will be taken to be the free quark decay rate; now $(\delta m)^{3} L_{\text {free }}(|\vec{q}|=$ $\delta m)$, as well as $\frac{d}{d|\vec{q}|}\left(|\vec{q}|^{4} L_{\text {free }}(|\vec{q}|)\right)(|\vec{q}|=\delta m)$, are of the order of the free quark decay rate (with our choice $L_{\text {free }}(|\vec{q}|=\delta m) \propto(\delta m)^{2}$ ).

Then, one can first observe that in fact not only all the terms written in eq. (29), (30) have a relative power $1 / m_{Q}^{2}$, but that they are more precisely of relative order $m_{d} \delta m / m_{b}^{2}$ at most ; terms of relative order $(\delta m)^{2} / m_{b}^{2}$ are already cancelled. This will be obtained more generally thanks to Bjorken sum rule. Now, the term of relative order $m_{d} \delta m / m_{b}^{2}$ encountered in the r.h.s. of the first integral (29) is cancelled by the first term in the r.h.s. of the second integral (30), just using Voloshin sum rule (19), i.e. $\Delta \tau^{2}=m_{d} / 2$. All the remaining contributions are of the type $(\delta m)^{5} \frac{1}{R^{2} m_{b}^{2}}$. We can evaluate them readily and find them to cancel too for the particular choice made for $L\left(q^{0},|\vec{q}|\right)$. Finally :

$$
\begin{aligned}
\delta \Gamma_{I}= & \int_{|\vec{q}|_{\text {max }, \text { free }}}^{|\overrightarrow{\vec{q}}|_{\text {max }}} \quad d|\vec{q}||\vec{q}|^{2} L_{\text {free }}(|\vec{q}|)- \\
& \int_{|\vec{q}|_{\text {max }, 1}}^{|\vec{q}|_{\text {max }}} \quad d|\vec{q}||\vec{q}|^{2} L_{\text {free }}(|\vec{q}|) \rho^{2} \frac{|\vec{q}|^{2}}{m_{b}^{2}} \simeq 0
\end{aligned}
$$

It must be emphasized that the cancellation can occur because the difference between $|\vec{q}|_{\text {max }, n}$ and $|\vec{q}|_{\text {max, free }}$ is changing sign between the ground state and the excitations. With our assumption $\Delta \ll \delta m$, one has $|\vec{q}|_{\text {max }, 1}<|\vec{q}|_{\text {max }, \text { free }}<|\vec{q}|_{\text {max }, 0}$

- Contribution II. It is also obvious that it contains already a power $\frac{1}{m_{b}^{2}}$. On factorising $(\delta m)^{5}$, one sees that $\frac{m_{d} \delta m}{m_{b}^{2}}$ terms are present in the first integral (second term of the bracket in the integrand) : $\left.\int_{0}^{|\vec{q}|_{\max , 0}} d q q^{2}\left(6 \delta m \frac{m_{d} q^{2}}{2 m_{b}^{2}}\right)\right)$ and in the second one (first term of the bracket in the integrand) : $\left.\int_{0}^{|\vec{q}|_{\max , 0}} d q q^{2}\left(6 \delta m(-\Delta)\left(\tau^{2} \frac{|\overrightarrow{\mid}|^{2}}{m_{b}^{2}}\right)\right)\right)$, the rest being smaller. It is easily seen that these $\frac{m_{d} \delta m}{m_{b}^{2}}$ terms cancel at this order, just using Voloshin sum rule $\Delta \tau^{2}=m_{d} / 2$, to leave a smaller contribution, which is only of order $\frac{1}{R^{2} m_{b}^{2}}$; the latter is found by performing a limited expansion of the integrand as above eq. (29) (the 
interval is once more $\mathcal{O}(\Delta))$, in powers of $\frac{\Delta}{\delta m}$ :

$$
3 \frac{m_{d} \delta m}{m_{b}^{2}} \int_{|\vec{q}|_{\max , 1}}^{|\vec{q}|_{\max , 0}} d|\vec{q}||\vec{q}|^{4} \simeq 3(\delta m)^{5} \frac{1}{R^{2} m_{b}^{2}}
$$

The other terms in the integrals are already manifestly of this order, and one ends with :

$$
\delta \Gamma_{I I}=\frac{9}{5}(\delta m)^{5} \frac{1}{R^{2} m_{b}^{2}}
$$

This result has been checked by a systematic expansion using Mathematica.

Finally, with $\Gamma_{\text {free }} \simeq \frac{4}{5}(\delta m)^{5}$ :

$$
\epsilon=\frac{\Gamma_{0}+\Gamma_{1}-\Gamma_{\text {free }}}{\Gamma_{\text {free }}} \simeq \frac{\frac{9}{5}(\delta m)^{5} \frac{1}{R^{2} m_{b}^{2}}}{\frac{4}{5}(\delta m)^{5}}=\frac{9}{4} \frac{1}{R^{2} m_{b}^{2}}
$$

Let us reinsist that it is of the order expected from OPE, unlike terms of the type $\frac{(\delta m)^{2}}{m_{b}^{2}}$ or $\frac{m_{d} \delta m}{m_{b}^{2}}$, which duely cancel, as has been shown.

\section{Relative magnitude of Isgur contribution}

- Let us now return briefly to the very discussion raised by ref. [1]. One could be worried why it is found there some duality violating effect, while we do not. The contradiction is only apparent. The answer seems to be that in totally integrated widths, the effect considered in [1] is finally relatively small parametrically with respect to the ones we have considered. Let us show that. The mismatch near zero recoil considered in [1] is the integral of the ground state contribution over $w_{0}(t)=\frac{m_{B}^{2}+m_{D}^{2}-t}{2 m_{B} m_{D}}$ between $w_{0}(t=$ $\left.\left(m_{B}-m_{D}\right)^{2}\right)=1$ and the threshold for the excited state production $w_{0}\left(t=\left(m_{B}-m_{D^{* *}}\right)^{2}\right)$ ( the variable $w$ for the ground state contribution is considered as a function of $t, w_{0}(t)$ ). Let us pass through the variable $\vec{q}$, which is more adapted to the NR problem, and denote as $|\vec{q}|_{n}(t)$ the value of $|\vec{q}|$ which corresponds to some $t$ for a state $n$; the total ground state contribution can be decomposed into two parts:

$$
\begin{aligned}
& \Gamma_{0} \simeq \int_{|\vec{q}|_{0}\left(t=\left(m_{B}-m_{D}\right)^{2}\right)}^{|\vec{q}|_{0}(t=0)} d|\vec{q}||\vec{q}|^{2} L_{n=0}(|\vec{q}|)\left(1-\rho^{2} \frac{|\vec{q}|^{2}}{m_{b}^{2}}\right)= \\
& \int_{|\vec{q}|_{0}\left(t=\left(m_{B}-m_{D}\right)^{2}\right)}^{|\vec{q}|_{0}(t=0)} d|\vec{q}||\vec{q}|^{2} L_{n=0}(|\vec{q}|)-\int_{|\vec{q}|_{0}\left(t=\left(m_{B}-m_{D}\right)^{2}\right)}^{|\vec{q}|_{0}(t=(0)} d|\vec{q}||\vec{q}|^{2} L_{n=0}(|\vec{q}|)\left(\rho^{2} \frac{|\vec{q}|^{2}}{m_{b}^{2}}\right)
\end{aligned}
$$

In the infinite mass limit, $m_{B}-m_{D^{* *}} \simeq m_{B}-m_{D} \simeq m_{b}-m_{c}$ and the functions $|\vec{q}|_{0}(t)$ and $|\vec{q}|_{1}(t)$, as well as $|\vec{q}|_{\text {free }}(t)$, become identical, and the functions $L\left(q^{0}, \vec{q}^{2}\right)$ become also identical for all states. Then, the first contribution equates the free quark decay rate, while the second one :

$$
\delta \Gamma_{0} \simeq \int_{|\vec{q}|_{0}\left(t=\left(m_{B}-m_{D}\right)^{2}\right)}^{|\vec{q}|_{0}(t=0)} d|\vec{q}||\vec{q}|^{2} L_{n=0}(|\vec{q}|)\left(-\rho^{2} \frac{|\vec{q}|^{2}}{m_{b}^{2}}\right)
$$


is exactly cancelled by the excited state contribution :

$$
\Gamma_{1} \simeq \int_{|\vec{q}|_{1}\left(t=\left(m_{B}-m_{D^{* *}}\right)^{2}\right)}^{|\vec{q}|_{1}(t=0)} d|\vec{q}||\vec{q}|^{2} L_{n=1}(|\vec{q}|) \tau^{2} \frac{|\vec{q}|^{2}}{m_{b}^{2}},
$$

due to Bjorken sum rule. Whence duality. However, when quark masses are finite, there is a small part of the integral (36) which is uncancelled, in spite of the Bjorken sum rule, by the corresponding excited state contribution, in particular because $t=\left(m_{B}-m_{D^{* *}}\right)^{2}$ now differs from $t=\left(m_{B}-m_{D}\right)^{2}$. We estimate the mismatch as :

$$
\delta \Gamma \simeq \int_{|\vec{q}|_{0}\left(t=\left(m_{B}-m_{D}\right)^{2}\right)}^{|\overrightarrow{\mid}|_{0}\left(t=\left(m_{B}-m_{D^{*}}\right)^{2}\right)} d|\vec{q}||\vec{q}|^{2} L_{n=0}(|\vec{q}|)\left(-\rho^{2} \frac{|\vec{q}|^{2}}{m_{b}^{2}}\right) .
$$

In this calculation, following [1], we disregard all other sources of difference, in particular the fact that the leptonic tensor functions are no more equal, and neither are the functions $|\vec{q}|_{n}(t)$ for $n=0$ and $n=1$ respectively, and that also the first contribution in 35 no longer equates the free quark decay rate. Then, our point is that this mismatch of total widths is very small with respect to the terms we have retained. Indeed, the integral runs over a small part of the phase space, but in addition the integrand is much smaller near zero recoil, where the mismatch takes place, first because of the leptonic factor $L\left(q^{0}, \vec{q}^{2}\right)=$ $3\left(q^{0}\right)^{2}-|\vec{q}|^{2}$, second because of the factor $\left(-\rho^{2} \frac{|\vec{q}|^{2}}{m_{b}^{2}}\right)$. Since $L\left(q^{0}, \vec{q}^{2}\right)=3\left(q^{0}\right)^{2}-|\vec{q}|^{2}$, using $|\vec{q}|_{0}\left(t=\left(m_{B}-m_{D^{* *}}\right)^{2}\right) \simeq \sqrt{\frac{2 \Delta}{\delta m}}|\vec{q}|_{0, \max }$ :

$$
\delta \Gamma \simeq \frac{\rho^{2}}{m_{b}^{2}} 3(\delta m)^{2} \frac{\left(\frac{2 \Delta}{\delta m}\right)^{\frac{5}{2}}}{5}|\vec{q}|_{0, \text { max }}^{5},
$$

and, relative to the free quark decay rate (i.e. contribution to $\epsilon$ ) :

$$
\frac{\delta \Gamma}{\Gamma_{\text {free }}} \simeq \frac{\rho^{2}}{m_{b}^{2}} \frac{3}{4}(\delta m)^{2}\left(\frac{2 \Delta}{\delta m}\right)^{\frac{5}{2}}
$$

which is parametrically small, because of the factor $\left(\frac{2 \Delta}{\delta m}\right)^{\frac{5}{2}}$ (since $\Delta \ll \delta m$ in the SV limit). In fact, in our calculation we have not retained such terms.

Numerically too, we find it very small, with real physical masses. It is true, as noticed in ref. [1], that numerically the region of Dalitz plot which is concerned is physically not very small, because one is far from the SV limit; with our approximative formula, we find around $20 \%$ of the free decay rate in this region of phase space, not far from the $30 \%$ estimated in ref. [1] ; but the factors considered above nevertheless combine to yield a very small effect for $\frac{\delta \Gamma}{\Gamma_{f r e e}}$, around $10^{-3} \rho^{2}$. This is due to the fact that the factor $\left(-\rho^{2} \frac{|\vec{q}|^{2}}{m_{b}^{2}}\right)$ is very small in this region of phase space.

\section{Conclusion}

Stimulated by the worries raised by N. Isgur, we have noticed mismatches between the sum of exclusive decays and the free quark total decay rate, which, considered separately, could convey the impression that quark hadron duality between total widths is violated at order $\delta m / m_{b}^{2}$, because all these mismatches are of this order. Let us recapitulate them : 
1) The upper limit in terms of $|\vec{q}|$ (corresponding to $t=0$ ) of the integrals for the ground state and the excited states contributions do not coincide. Therefore, the contributions from the falloff of ground state and rise of excited states do not cancel near $|\vec{q}|_{\max }(t=0)$.

2) The upper limit in $|\vec{q}|$ of the integrals for the ground state contribution and the free quark decay do not coincide for similar reasons.

3) The leptonic tensors of the various contributions are different, because the function $q^{0}(|\vec{q}|)$ depends on the transition considered.

At order $\left.\mathcal{O}\left(\frac{\delta m}{m_{b}^{2}}\right), 1\right)$ and 2) cancel between each other, while 3) has a zero net effect, by internal cancellation of the differences of leptonic tensors, when integrated (taking into account the difference in upper limits of integration in $|\vec{q}|$, near maximum recoil, is once more necessary).

It must be emphasized that even in this simple model and in the SV limit, it is by no means trivial to check duality, because the check requires to take into account detailed effects, such as the dependence of ground state binding energy on the heavy quark masses through their different radii, which itself reflects the flavor independence of the quark potential, etc...

In both cases, the cancellation occurs because of Voloshin sum rule. The consideration of it is absolutely necessary, in addition to Bjorken one, to demonstrate duality of total widths through summation of exclusive states at subleading order. Note that, if we have an independent mean to demonstrate duality, for example by a rigourous demonstration of OPE to the required order, we can use the result on the sum of exclusive states, on the reverse, to demonstrate these sum rules.

\section{Acknowledgements}

A.L.Y., L.O., O.P. and J.-C. R. acknowledge partial support from the EEC-TMR Program, contract N. CT 98-0169.

\section{References}

[1] Nathan Isgur, hep-ph/9811377, Phys.Lett. B448 (1999) 111-118.

[2] M. Shifman, M.B. Voloshin, Sov. J. Nucl. Phys. 47 (1988) 511.

[3] A. Le Yaouanc, D. Melikhov, V. Morenas, L. Oliver, O. Pène and J.-C. Raynal, hep-ph/0003087, to be published in Phys. Lett.

[4] I. Bigi, M. Shifman, N. G. Uraltsev and A. Vainshtein Phys.Rev. D52 (1995) 196.

[5] A. Le Yaouanc, D. Melikhov, V. Morenas, L. Oliver, O. Pène and J.-C. Raynal, LPT 00-30, HD-THEP-00-13, hep-ph/0004246.

[6] A. Le Yaouanc, D. Melikhov, V. Morenas, L. Oliver, O. Pène and J.-C. Raynal, to appear. 